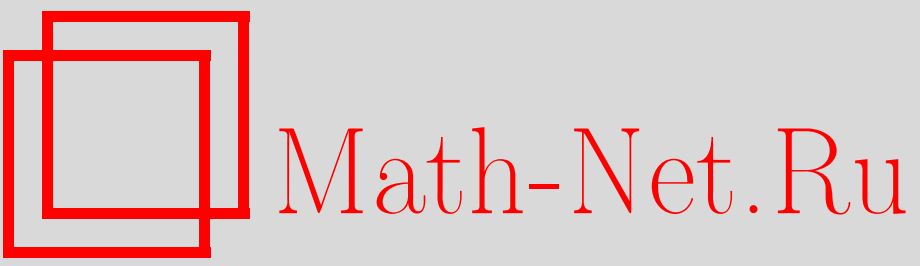

Н. А. Шананин, K продолжению ростков решений уравнений второго порядка, Итоги науки и техн. Сер. Соврем. мат. и ее прил. Темат. обз., 2021, том 192, 150-154

DOI: https://doi.org/10.36535/0233-6723-2021-192-150-154

Использование Общероссийского математического портала Math-Net.Ru подразумевает, что вы прочитали и согласны с пользовательским соглашением

http: //www . mathnet.ru/rus/agreement

Параметры загрузки :

IP : 54.197 .217 .227

26 апреля 2023 г., 15:16:59 


\title{
К ПРОДОЛЖЕНИЮ РОСТКОВ РЕШЕНИЙ УРАВНЕНИЙ ВТОРОГО ПОРЯДКА
}

\author{
(c) 2021 г. $\quad$ Н. А. ШАНАНИН
}

\begin{abstract}
АннотАция. Для решений линейных дифференциальных уравнений второго порядка с вещественными коэффициентами и знакоопределенным старшим символом получены теоремы об однозначном продолжении ростков вдоль кривых.
\end{abstract}

Ключевъе слова: росток решения, однозначное продолжение.

\section{ON THE CONTINUATION OF GERMS OF SOLUTIONS OF SECOND-ORDER EQUATIONS}

\author{
(c) 2021 N. A. SHANANIN
}

Abstract. For solutions of second-order linear differential equations with real coefficients and a signdefinite leading symbol, theorems on the unique continuation of germs along curves are obtained.

Keywords and phrases: germ of solution, unique continuation.

AMS Subject Classification: 35A01

Статья дополняет работы автора $[5,6]$ по однозначному продолжению ростков решений дифференциальных уравнений вдоль кривых. В статье рассматривается класс операторов второго порядка с вещественными бесконечно дифференцируемыми коэффициентами, со знакоопределенным старшим символом и двукратным характеристическим множеством. Терминология и обозначения соответствуют принятым в $[2,3]$.

1. Основные результаты. Пусть $\Omega$ - открытое множество в $\mathbb{R}^{n}$ и

$$
P=\sum_{l, k=1}^{n} a_{l, k}(x) \partial_{x_{l}} \partial_{x_{k}}+\sum_{k=1}^{n} b_{k}(x) \partial_{x_{k}}+c(x), \quad x \in \Omega,
$$

- дифференциальный оператор с вещественными бесконечно дифференцируемыми коэффициентами, $a_{l, k}(x)=a_{k, l}(x), l, k=1,2, \ldots, n$. Система координат $x$ на множестве $\Omega$ индуцирует систему координат $(x, \partial / \partial x)$ на кокасательном пространстве $T^{*}(\Omega)$, определяя изоморфизм кокасательного пространства на произведение $\Omega \times \mathbb{R}^{n}$. Везде ниже в формулировках используется указанная система координат. Предположим, что выполнены следующие условия:

(i) в каждой точке $x \in \Omega$ главный символ

$$
p_{2}(x, \xi)=-\sum_{l, k=1}^{n} a_{l, k}(x) \xi_{l} \xi_{k}, \quad(x, \xi) \in T^{*}(\Omega),
$$

оператора $P$ является знакоопределенной квадратичной формой на слоях кокасательного расслоения $T_{x}^{*}(\Omega)$; 
(ii) в каждой точке $x \in \Omega$ ядро $\mathcal{K}_{x}(P) \subset T_{x}^{*}(\Omega)$ одномерно;

(iii) $\mathcal{L}(P)$ - инволютивная дифференциальная система;

(iv) субглавный символ

$$
p_{\text {sub }}(x, \xi)=i \sum_{k=1}^{n}\left(\sum_{l=1}^{n} \frac{\partial a_{l, k}}{\partial x_{l}}(x)+b_{k}(x)\right) \xi_{k}
$$

оператора $P$, определенный в точках $(x, \xi)$, принадлежащих характеристическому множеству, отличен от нуля при $\xi \in \mathcal{K}_{x}(P)$.

Из канонического представления квадратичной формы следует, что при выполнении условия (i) характеристическое множество оператора $\operatorname{Char}(P)=\left\{(x, \xi) \mid p_{2}(x, \xi)=0, \xi \neq 0\right\}$ состоит из множества двукратно-характеристических (см. определение в [2,3]) точек оператора:

$$
\left\{(x, \xi) \in \Omega \times\left(\mathbb{R}^{n} \backslash\{0\}\right) \mid p_{2}(x, \xi)=0, d_{\xi} p_{2}(x, \xi)=0\right\} .
$$

Обозначим через $\mathcal{K}_{x}(P)$ ядро в точке $x \in \Omega$ квадратичной по $\xi \in \mathbb{R}^{n}$ формы $p_{2}(x, \xi)$ :

$$
\mathcal{K}_{x}(P)=\left\{\xi \in \mathbb{R}^{n}: \sum_{l, k=1}^{n} a_{l, k}(x) \eta_{l} \xi_{k} \forall \eta \in \mathbb{R}^{n}\right\} .
$$

Для операторов $P$, удовлетворяющего условию (i), включение $(x, \xi) \in \operatorname{Char}(P)$ равносильно включению $\xi \in \mathcal{K}_{x}(P)$ и $\xi \neq 0$.

Из условия (ii) следует, что множество

$$
\mathcal{L}(P)=\left\{(x, \tau) \in T(\Omega) \mid \xi(\tau)=0 \forall \xi \in \mathcal{K}_{x}(P)\right\}
$$

образует $(n-1)$-мерную дифференциальную систему в касательном пространстве $T(\Omega)$. Гладкую кривую $\Gamma=\{x(t) \in \Omega \mid t \in(a, b) \subset \mathbb{R}\}$ называют интегральной кривой для дифференциальной системы $\mathcal{L}(P)$, если в каждой точке $x \in \Gamma$ касательный к кривой вектор $\tau_{x}$ удовлетворяет условию $\tau_{x} \in \mathcal{L}(P)$.

При выполнении условия (iii) через каждую точку $x$ множества $\Omega$ проходит интегральное подмногообразие $\Omega_{x, \mathcal{L}(P)}$ дифференциальной системы $\mathcal{L}(P)$ максимальной размерности $\operatorname{dim} \Omega_{x, \mathcal{L}(P)}=$ $n-1$.

Говорят, что линейный дифференциальный оператор $P$ является гипоэллиптическим на множестве $\Omega$, если для любого открытого подмножества $V \subset \Omega$ из включений $u(x) \in \mathcal{D}^{\prime}(\Omega)$ и $P u(x) \in C^{\infty}(V)$ следует включение $u(x) \in C^{\infty}(V)$.

Теорема 1. Если оператор $P$ вида (1) удовлетворяет условиям (i)-(iv), то он является гипоэллиптическим на множестве $\Omega$.

Говорят, что ростки функций $u^{j}(x) \in \mathcal{D}^{\prime}(\Omega), j=1,2$, равны в точке $x^{0} \in \Omega$ и пишут $u_{x^{0}}^{1} \cong$ $u_{x^{0}}^{2}$, если существует открытая окрестность $V \subset \Omega$ этой точки, в которой $u^{1}(x)=u^{2}(x)$, т.е. для любой основной функции $\varphi(x) \in \mathcal{D}(\Omega)$ с носителем supp $\varphi(x) \subset V$ выполняется равенство $\left\langle u^{1}, \varphi\right\rangle=\left\langle u^{2}, \varphi\right\rangle$.

Теорема 2. Если оператор $P$ вида (1) удовлетворяет условиям (i)-(iv), то для любых $u^{1}(x)$ $u u^{2}(x) \in \mathcal{D}^{\prime}(\Omega)$ из равенства ростков $u_{x^{0}}^{1} \cong u_{x^{0}}^{2}$ в точке $x^{0}$ вытекает равенство $u_{x}^{1} \cong u_{x}^{2}$ во всех точках связной компоненты $\Pi_{x^{0}}$ множества

$$
\Omega_{x^{0}, \mathcal{L}(P)} \cap\left\{x \mid\left(P u^{1}\right)_{x} \cong\left(P u^{2}\right)_{x}\right\},
$$

содержащей точку $x^{0}$.

Пусть $\Gamma=\{x(t) \in \Omega \mid t \in(a, b) \subset \mathbb{R}\}$ - непрерывная кривая. Будем говорить, что ростки решений дифференциального уравнения

$$
P u=f
$$


однозначно продолжсаются вдоль $\Gamma$, если для любых двух обобщенных функций $u^{1}(x)$ и $u^{2}(x) \in$ $\mathcal{D}^{\prime}(\Omega)$, удовлетворяющих условию $P u_{x}^{1} \cong P u_{x}^{2}$ при всех $x \in \Gamma$, из равенства ростков $u_{x^{*}}^{1} \cong u_{x^{*}}^{2}$ в некоторой точке $x^{*} \in \Gamma$ следует, что $u_{x}^{1} \cong u_{x}^{2}$ во всех точках $x \in \Gamma$.

Как следствие из теоремы 2 получаем теорему об однозначном продолжении ростков решений дифференциального уравнения вдоль интегральных кривых дифференциальной системы, ассоциированной со старшим символом.

Теорема 3. Если оператор $P$ вида (1) удовлетворяет условиям (i)-(iv), то ростки решений дифференциального уравнения $P u=f$ однозначно продолюаются вдоль интегральных кривых дифберенииальной системы $\mathcal{L}(P)$.

Отметим, что утверждение теоремы можно формально усилить, заменив в его формулировке множество интегральных кривых на множество непрерывных кривых, содержащихся в интегральных слоях $\Omega_{x, \mathcal{L}(P)}$ дифференциальной системы $\mathcal{L}(P)$.

2. Доказательства теорем. Начнем с построения локальной замены координат к виду анизотропного оператора со взвешенными производными. В результате замены координат $x=x(y)$ старший символ оператора (1) преобразуется по хорошо известному закону

$$
p_{2}(y, \eta)=\left.p_{2}(x, J(x) \eta)\right|_{x=x(y)},
$$

где $J(x)$ - матрица Якоби. При выполнении наложенных условий замену координат можно подобрать так, что оператор будет преобразован к специальному виду.

Лемма 1. Предположим, что оператор Р вида (1) удовлетворяет условиям (i)-(iii). Тогда для любой точки $x^{0} \in \Omega$ найдется локальная система координат $y=\left(y_{1}(x), \ldots, y_{n-1}(x), \varphi(x)\right)$, определенная в некоторой окрестности $U$ точки $x^{0}$, в которой оператор имеет следующий вид:

$$
P=\sum_{l, k=1}^{n-1} \widetilde{a}_{l, k}(y) \partial_{y_{l}} \partial_{y_{k}}+\sum_{k=1}^{n-1} \widetilde{b}_{k}(y) \partial_{y_{k}}+\left.\frac{1}{i} p_{\text {sub }}\left(x, \varphi_{x}(x)\right)\right|_{x=x(y)} \partial_{y_{n}}+\widetilde{c}(y),
$$

где $y_{n}=\varphi(x)$.

Доказательство. Из условий (ii) и (iii) следует, для любой точки $x^{0} \in \Omega$ найдется локальная система координат $\left(y_{1}(x), \ldots, y_{n-1}(x), y_{n}(x)\right)$, определенная в окрестности $U$ рассматриваемой точки, в которой интегральные многообразия дифференциальной системы задаются уравнениями вида $y_{n}=$ const. В каждой точке $x \in U$ функция $\varphi(x)=y_{n}(x)$ удовлетворяет включению $\varphi_{x}(x) \in$ $\mathcal{K}_{x}(P)$, равносильному системе дифференциальных уравнений

$$
\sum_{k=1}^{n} a_{j, k}(x) \varphi_{x_{k}}(x)=0, \quad j=1,2, \ldots, n .
$$

Отсюда, во-первых, вытекает, что $\widetilde{a}_{j, n}(y)=\widetilde{a}_{n, j}(y)=0$ при всех $j=1,2, \ldots, n$, и, кроме того,

$$
\sum_{j, k=1}^{n} a_{j, k}(x) \varphi_{x_{j}, x_{k}}(x)=-\sum_{j, k=1}^{n} \frac{\partial a_{j, k}}{\partial x_{j}}(x) \varphi_{x_{k}}(x) .
$$

Из полученных равенств, очевидно, следует координатное представление (3).

Обозначим через $y^{0}$ и $\widetilde{U}$ образы точки $x^{0}$ и ее окрестности $U$, индуцированные отображением $x \rightarrow y(x)$ соответственно. В операторе (3) произведем взвешивание производных. Операциям дифференцирования

$$
D_{j}=\frac{1}{i} \frac{\partial}{\partial_{y_{j}}}
$$

по переменным $y_{j}$ при $j=1, \ldots, n-1$ присвоим веса, равные 1 , а дифференцированию

$$
D_{n}=\frac{1}{i} \frac{\partial}{\partial_{y_{n}}}
$$


по переменной $y_{n}$ - вес 2. Взвешенный порядок рассматриваемого оператора равен 2, причем его взвешенный главный символ имеет вид

$$
p_{2, w}(y, \eta)=-\sum_{l, k=1}^{n-1} \widetilde{a}_{l, k}(y) \eta_{l} \eta_{k}+\widetilde{a}_{n}(y) \eta_{n}
$$

где $\widetilde{a}_{n}(y)=\left.p_{\mathrm{sub}}\left(x, \varphi_{x}(x)\right)\right|_{x=x(y)}$. В силу условия (iv) коэффициент $\widetilde{a}_{n}(y)$ отличен от нуля и принимает чисто мнимые значения.

В соответствии со взвешиванием, используя координаты $(y, \eta)$, определим в слоях кокасательного расслоения $T^{*}(U)\left(\cong \widetilde{U} \times \mathbb{R}_{\eta}^{n}\right)$ действие мультипликативной группы $\mathbb{R}_{+}$:

$$
\lambda \circ\left(x ; \eta_{1}, \ldots, \eta_{n-1}, \eta_{n}\right)=\left(x ; \lambda \cdot \eta_{1}, \ldots, \lambda \cdot \eta_{n-1}, \lambda^{2} \cdot \eta_{n}\right), \quad \lambda \in \mathbb{R}_{+}
$$

Множество $\Gamma \subset \mathbb{R}_{\eta}^{n}$ называют квазиконическим, если из включения $\eta \in \Gamma$ следует, что $\lambda$ о $\eta \in \Gamma$ при всех $\lambda \in \mathbb{R}_{+}$. Введем в рассмотрение весовую функцию

$$
|\eta|_{w}=\left(\sum_{k=1}^{n-1} \eta_{k}^{2}+\left|\eta_{n}\right|\right)^{1 / 2}
$$

Говорят, что точка $\left(y^{*}, \eta *\right) \in \widetilde{U} \times \mathbb{R}_{\eta}^{n}$ не принадлежит взвешенному волновому фронту $W F_{w}(u)$ обобщенной функции $u(y) \in \mathcal{D}^{\prime}(\widetilde{U})$ и пишут $\left(y^{*}, \eta^{*}\right) \notin W F_{w}(u)$, если существуют такие окрестность $U_{y^{*}} \subset \widetilde{U}$ точки $y^{*}$ и квазиконическая окрестность $\Gamma_{\eta^{*}}$ точки $\eta^{*}$, что для любых основной функции $v(y) \in \mathcal{D}\left(U_{y^{*}}\right)$ и вещественного числа $N$ найдется константа $C$, с которой для всех $\eta \in \Gamma_{\eta^{*}}$ выполняется оценка

$$
|(u(y), v(y) \exp (i\langle y, \eta\rangle))| \leqslant C|\eta|^{-N}
$$

где $\langle y, \eta\rangle=y_{1} \eta_{1}+\ldots+y_{n-1} \eta_{n-1}+y_{n} \eta_{n}$. Очевидно, $u(y) \in C^{\infty}(\widetilde{U})$, если и только если $W F_{w}(u)=\varnothing$. Множество

$$
\operatorname{Char}(P)=\left\{(y, \eta) \in \widetilde{U} \times\left(\mathbb{R}_{\eta}^{n} \backslash\{0\}\right) \mid p_{2, w}(y, \eta)=0\right\}
$$

характеристических точек анизотропного оператора (3) состоит из точек, в которых

$$
-\sum_{l, k=1}^{n-1} \widetilde{a}_{l, k}(y) \eta_{l} \eta_{k}=0 \text { и } \widetilde{a}_{n}(y) \eta_{n}=0 .
$$

Из условий (i) и (iv) следует, что оно пусто.

2.1. Доказательство теоремы 1. Предположим, что $u(x) \in \mathcal{D}^{\prime}(\Omega)$ и $P u(x) \in C^{\infty}(V)$. Достаточно доказать, что для любой точки $x^{0} \in V$ найдется окрестность, в которой функция $u$ является бесконечно дифференцируемой. В силу леммы 1 для $x^{0}$ найдется окрестность $U$ и определенная в ней локальная замена координат, в которой оператор $P$ имеет вид анизотропного оператора (3). Поскольку при сделанных предположениях множество характеристических точек оператора (3) является пустым, то в силу [1, теорема 1] для анизотропного волнового фронта функции $u$ выполняется включение

$$
W F_{w}(u) \cap\left(\widetilde{U} \times \mathbb{R}_{\eta}^{n}\right) \subset W F_{w}(P u) \cap\left(\widetilde{U} \times \mathbb{R}_{\eta}^{n}\right)
$$

Так как $P u(x) \in C^{\infty}(U \cap V)$, то $\left.W F_{w}(P u) \cap(\widetilde{(U \cap V}) \times \mathbb{R}_{\eta}^{n}\right)=\varnothing$. Вследствие включения отсюда имеем $\left.W F_{w}(u) \cap(\widehat{(U \cap V}) \times \mathbb{R}_{\eta}^{n}\right)=\varnothing$ и, следовательно, $u \in C^{\infty}(U \cap V)$. Теорема доказана. 
2.2. Доказательство теоремы 2. Положим $w=u^{1}(x)-u^{2}(x)$. Тогда по условию теоремы $w_{x^{0}} \cong$ 0 и $P w=0$ в некоторой окрестности $\Omega_{1}$ связной компоненты $\Pi_{x^{0}}$. В силу теоремы $1 w(x) \in$ $C^{\infty}\left(\Omega_{1}\right)$. Если утверждение теоремы не верно, то найдется точка $x^{1} \in \Pi_{x^{0}}$, в которой $w_{x^{1}} \nsucceq 0$. Пусть $\Gamma=\left\{x(t) \in \Pi_{x^{0}} \mid t \in[0,1]\right\}$ - гладкий путь, соединяющий точки $x^{0}=x(0)$ и $x^{1}=x(1)$. Обозначим через

$$
t^{*}=\sup \left\{t \in[0,1] \mid w_{x(s)} \cong 0 \text { при всех } s<t\right\} .
$$

Поскольку $w_{x^{1}} \nsucceq 0$, то $t^{*} \in(0,1]$. Положим $x^{*}=x\left(t^{*}\right)$. В силу леммы 1 в некоторой окрестности $U$ точки $x^{*}$ заменой координат оператор $P$ можно привести к виду анизотропного оператора (3). Сужая при необходимости окрестность точки $x^{*}$, можно добиться того, что пересечение $U \cap$ $\Pi_{x^{*}}$ будет связным и содержать точку, в окрестности которой $w(x)=0$, функция $w(x)$ будет бесконечно дифференцируемой в $U$ и удовлетворять уравнению $P w(x)=0$.

Для завершения доказательства воспользуемся теоремой 4.4 из [4], предварительно проверив выполнение ее условий. В обозначениях [4] $\eta^{\prime}=\left(\eta_{1}, \ldots, \eta_{n-1}\right)$ и $\eta^{\prime \prime}=\eta_{n}$. Поскольку характеристическое множество анизотропного оператора пусто, то оператор является $\eta^{\prime}$-квазиэллиптическим. Далее при всех неколлинеарных $\left(\eta^{\prime}, 0\right)$ и $\left(\zeta^{\prime}, \zeta_{n}\right)$ квадратный многочлен

$$
p_{2, w}\left(y, z \eta^{\prime}+\zeta^{\prime}, \zeta_{n}\right)=-z^{2}\left\langle\widetilde{A}(y) \eta^{\prime}, \eta^{\prime}\right\rangle-2 z\left\langle\widetilde{A}(y) \eta^{\prime}, \zeta^{\prime}\right\rangle-\left\langle\widetilde{A}(y) \zeta^{\prime}, \zeta^{\prime}\right\rangle+\widetilde{a}_{n}(y) \zeta_{n},
$$

где

$$
\left\langle\widetilde{A}(y) \eta^{\prime}, \zeta^{\prime}\right\rangle=\sum_{l, k=1}^{n-1} \widetilde{a}_{l, k}(y) \eta_{l}^{\prime}
$$

$\zeta_{k}^{\prime}$ не имеет вещественных корней. Кроме того, в силу условия (i) имеем

$$
\frac{\left\langle\widetilde{A}(y) \zeta^{\prime}, \zeta^{\prime}\right\rangle}{\left\langle\widetilde{A}(y) \eta^{\prime}, \eta^{\prime}\right\rangle}=\frac{p_{2, w}\left(y, \zeta^{\prime}, 0\right)}{p_{2, w}\left(y, \eta^{\prime}, 0\right)}>0
$$

для любого $\zeta^{\prime} \neq 0$. Отсюда следует, что дискриминант

$$
4\left(\left\langle\widetilde{A}(y) \eta^{\prime}, \zeta^{\prime}\right\rangle^{2}-\left\langle\widetilde{A}(y) \eta^{\prime}, \eta^{\prime}\right\rangle\left\langle\widetilde{A}(y) \zeta^{\prime}, \zeta^{\prime}\right\rangle-\left\langle\widetilde{A}(y) \eta^{\prime}, \eta^{\prime}\right\rangle \widetilde{a}_{n}(y) \zeta_{n}\right)
$$

многочлена $p_{2, w}\left(y, z \eta^{\prime}+\zeta^{\prime}, \zeta_{n}\right)$ отличен от нуля при всех $y \in U$ и неколлинеарных $\left(\eta^{\prime}, 0\right)$ и $\left(\zeta^{\prime}, \zeta_{n}\right)$ и, следовательно, кратных корней на этом множестве параметров многочлен не имеет. Теперь, применяя [4, предложение 4.1 и теорема 4.4 ], получаем, что $\operatorname{supp} w \cap\left(U \cap \Pi_{x^{*}}\right)=\varnothing$, т.е. $w_{x} \cong 0$ в точках $x \in U \cap \Pi_{x^{*}}$ и, в частности, $w_{x^{*}} \cong 0$. Последнее противоречит выбору точки $x^{*}$. Теорема доказана.

\section{СПИСОК ЛИТЕРАТУРЫ}

1. Грушин В. В., Шананин Н. А. Некоторые теоремы об особенностях решений дифференциальных уравнений со взвешенными главными символами// Мат. сб. - 1977. - 103, № 1. - С. 37-51.

2. Трев $\Phi$. Введение в теорию псевдо-дифференциальных операторов и интегральных операторов Фурье. - М.: Мир, 1984.

3. Хермандер Л. Анализ линейных дифференциальных операторов с частными производными. - М.: Мир, 1984.

4. Шананин H. А. Об однозначном продолжении решений дифференциальных уравнений со взвешенными производными// Мат. сб. - 2000. - 191, № 3. - С. 113-142.

5. Шананин Н. А. Об однозначном продолжении вдоль кривых ростков решений линейных дифференциальных уравнений с постоянными коэффициентами// Мат. заметки. - 2017. - 102, № 1. - С. 152-157.

6. Шананин H. А. Об однозначном продолжении ростков решений дифференциальных уравнений первого порядка вдоль кривых// Мат. заметки. - 2017. - 102, № 6. - С. 917-930.

Шананин Николай Алексеевич

Государственный университет управления, Москва

E-mail: nashananin@inbox.ru 